\title{
E-Marketplace for Semantic Web Services
}

\author{
Witold Abramowicz, Konstanty Haniewicz, Monika Kaczmarek, \\ and Dominik Zyskowski
}

\author{
Department of Information Systems, Poznan University of Economics Al. \\ Niepodleglosci 10, 60-967 Poznan, Poland \\ \{w.abramowicz,k.haniewicz,m.kaczmarek, d.zyskowski\}@kie.ae.poznan.pl
}

\begin{abstract}
Automation of processes is a crucial factor for enterprises operating within a modern collaborative business environment. In order to ensure flexible operations, companies tend to build their IT systems in accordance with the SOA paradigm and take advantage of the Semantic Web technologies. The mentioned tendency especially in case of cooperating organizations requires support for automated service discovery and fast integration of discovered artefacts. Currently, one can easily find several initiatives that aim at automation of already pointed tasks. As a part of this work, we analyze a number of different frameworks that implement, support and facilitate interactions inherent to Semantic Web services and indicate their shortcomings. Having completed this survey, we propose a general model of SWS e-marketplace taking into account all important aspects that a featured model should provide. In order to achieve this goal, a set of features provided by the surveyed frameworks is compiled with a set of additional traits that were not considered before. Moreover, the model is enriched with economical requirements driven by service providers' and service requesters' needs.
\end{abstract}

\section{Introduction}

Automation of enterprise processes is a crucial factor for enterprises operating within a modern collaborative business environment. In order to ensure flexible operations, companies tend to build their IT systems in accordance with the SOA paradigm and take advantage of the Semantic Web technologies 1. The mentioned tendency especially in case of cooperating organizations requires support for automated service discovery and fast integration of discovered artefacts.

As the conducted research and current initiatives showed, the service-oriented computing requires an infrastructure that provides a mechanism for coordinating between service requesters and providers [2. Such a coordination mechanism may take different forms. Various research initiatives were undertaken in order to provide a fully-fledged platform that would enable the automated interactions between service requesters and service providers. However, majority of the research concentrates on selected aspects of Semantic Web services (SWS) usage in the context of service orientation. The most active areas include service composition, discovery, description methods or contracting.

A. Bouguettaya, I. Krueger, and T. Margaria (Eds.): ICSOC 2008, LNCS 5364, pp. 271-285 2008.

(C) Springer-Verlag Berlin Heidelberg 2008 
Within this work we present an abstract model of SWS e-marketplace defined based on the observations of the SOA and Web services market evolution. We argue that the problem of Semantic Web services provisioning requires incorporation of business-oriented point of view and that an additional set of mechanisms should be taken into account in order to meet business expectations. Within our approach, we do not ignore the fact that SWS are also a kind of good that is provided and requested by some entities. Therefore, we pay special attention to a way of describing clients' needs and providers' services in order to assure possibly complete information on services offered. We also highlight the problem of service quality representation.

A reference model of a marketplace is given for the sake of clarity and building common understanding of concepts later discussed. The model is defined with aid of a set of crucial concepts that were designed to be straightforward and descriptive to convey all information needed for further reference. A study of adoption of the concepts enumerated is presented.

The article is structured as follows. First, a necessary introduction into a research domain and current trends is given, based on the experience and work done by scientists involved in various European and worldwide initiatives revolving around a notion of Web service and its semantically annotated counterpart. Then, a more detailed picture of the marketplace is drawn by taking into account initiatives centred on electronic commerce in general. This is of utmost importance for the work as it is impossible to imagine a working marketplace that does not implement a number of standards and features desired and expected to be in place. Core functionalities are later enhanced with ones that seem to be omitted either due to the concrete assumptions of reviewed initiatives or due to other unknown to the authors reasons. Further on, a presentation of reference model electronic marketplace is followed by a complete list of functionalities that characterize the featured electronic marketplace is given along with discussion of its soundness. The discussion of the soundness is set as a result of a comparison of other initiatives of electronic marketplaces. The article concludes in a summary of efforts and shows the directions of our future work.

\section{Open Service Marketplace and Research Roadmap}

As pinpointed by [3] the market of Web services evolves into a direction of the open Web services market, as depicted in 11. This vision is also supported by the research roadmap defined by European experts in the domain of service oriented computing [4] as well as current trends e.g. 1] or 41]. The mentioned vision and evolution concerns the different stages (i.e. layers) of SOA development as well as application of Semantic Web technologies to achieve automation of certain interactions.

Within the vision of extended SOA [3, three main layers may be distinguished. The first one focuses on atomic services, their descriptions and basic operations such as: publication, discovery, selection and binding; producing or utilizing their description. This layer constitutes the SOA foundation. It has already been 
implemented not only for the Web services but also for Semantic Web services (e.g. DIP'1 or ASG 2 ).

Upper layers provide an additional support required for service composition and service management. In the second layer, the composition of services requires an existence of additional functionalities, namely: coordination of the composite service execution, monitoring as well as conformance that are to ensure the integrity of the composite services and finally assurance of the quality of the composite service.

In the top layer, the organizations responsible for performing management functions (such as QoS assurance, overall maintenance etc.) are situated. They are called service operators, which may be service clients or composite service creators. The aim of the third layer is also to provide a support for open service marketplaces.

The authors [3] argue that the purpose of an open service market is to create opportunities for buyers and sellers to meet and make business electronically, or aggregate service supply/demand by offering added-value services and grouping the buying power. The scope of such a service marketplace would be limited only by the ability of enterprises to make the offer visible to other enterprises and establish industry-specific protocols to conduct business.

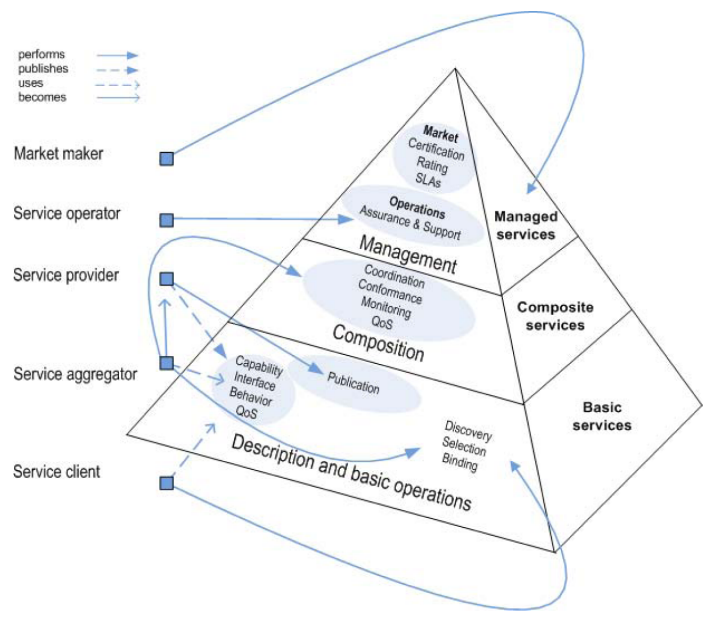

Fig. 1. Extended SOA 3

It may be argued that we are currently situated in the third layer of SOA development, however, the question appears what form such a marketplace should take and which functionalities it should offer. As the conducted research showed, the service-oriented computing requires an infrastructure that provides a mechanism for coordinating between service requesters and providers [2]. Such a

\footnotetext{
1 http://dip.semanticweb.org

2 http://asg-platform.org
} 
coordination mechanism may be implemented in various forms. Abstracting from the model that will be used, the platform where potential business partners can be discovered, prices can be ascertained and contracts may be signed is required.

The answer to the type of this platform may be found by reviewing the current initiatives and trends [1, 29]-38] and taking into account the already mentioned research 4. Based on current findings, we argue that the most appropriate architecture for inter-organisational collaboration should be an e-marketplace adopted to particular characteristics of SWS. Therefore, apart from typical SWS interactions like composition an additional focus is paid to industry community, business exchanges and institutional and governance aspects. The detailed description of SWS e-marketplace features is presented in the next section.

\section{Electronic Marketplace of SWS - Requirements}

Taking all of the already mentioned issues into account, it seems that the best form that the open service market may take is the semantics-based e-marketplace [17] 18 targeted at B2B interactions. As SWS are in fact the good that is may be traded on the ontology-based e-marketplace, thus, instead of a Web services e-marketplace a SWS e-marketplace should be addressed.

Bakos [8] defines an electronic marketplace as an inter-organizational information system that allows the participating buyers and sellers in some market to exchange information about process and product offerings. Other definitions stress that e-marketplaces are intermediaries that allow buyers and sellers to meet on an electronic platform that rests on the Internet infrastructure in order to exchange information about products/services (e.g. prices, specifications), conduct transactions online and adhere to other value-added services (e.g. settlement, distribution, integration, SCM) offered by the intermediary [9].

E-marketplace activity has been evolving from the early matchmaking models to more complex interactive and interconnected marketplaces. Following [10, four phases of e-marketplace evolution may be distinguished. It began with the transaction focus (the first phase) and evolved into the value-added marketplace that offers transaction support services (the second phase). In the third phase, the e-marketplace's services allow for not only information exchange but also for knowledge exchange facilitating cross-organizational collaboration. Finally, the ability to integrate the transaction exchange, the value-add services and the knowledge services moves the evolution of e-marketplaces into the fourth phase called Value Trust Networks (VTN).

In the recent years, e-marketplace proved to be sound solution to promote intra-organisational cooperation [1],12]13. Moreover, collaboration-oriented e-marketplaces are cited as an emerging approach to support online business-tobusiness transactions [14. As indicated in [15] the main goal of e-marketplaces in their formation phase was to bring different trading partners together. However, the requirements on e-marketplaces already increased within this phase. Companies demand additional features for lowering costs and for automation and optimization of their business processes. According to this, the aim for 
e-marketplaces is to offer more automation and value add services, such as offering services for initiation, fulfilment, and completion of trading transactions including shipment, payment and logistic services [15.

The research in the area of e-marketplaces proved that the utilization of ontologies facilitates the processes of e-marketplace, from matchmaking, recommendation, to negotiation [16 and helps to achieve the desired level of automation [15. In addition, ontology allows to solve some typical problems in e-marketplaces (see [16] for details). Taking all of the above issues in the account, it seems that the best form that the open service market may take is the ontology-based e-marketplace targeted at B2B interactions.

The Semantic Web services e-marketplace should incorporate most functionalities offered currently by online marketplaces like eBay, Amazon or auction portals [17]. Functionalities like discovery, personalization, payment, delivery, shipment tracking [19] are the must-have items on the functionality list of every modern web-based marketplace. The minimal set of functionalities that the SWS e-marketplace should support is as follows [14] :

- dynamic discovery of services and business processes,

- generation of reusable services and business processes,

- registering and advertising of available services and business processes in a proper structure.

However, we argue that the e-marketplace of Semantic Web services should posses also some additional mechanisms to meet specific requirements of the clients and providers. According to 32 , the platform should allow multiple buyers and sellers to trade simultaneously and ensure an immediate reaction in case a suitable counterpart is found. The mechanism should support trading of heterogeneous services. In fact, a meaningful matchmaking of orders should be realized by the market infrastructure to allow matching of services based on the semantics of an order instead of their syntactical representation. Furthermore, services may differ in their quality characteristics and their policies, e.g. a stock quote service by its quote time; a billing service by its age restriction. As such, the mechanism should support services with many attributes.

In order to meet these requirements, an e-marketplace of Semantic Web services needs to provide a unified view of services, standard business terminology and detailed descriptions of composite services. It is important to assure that clients and service providers use the same vocabulary and language to describe offered or requested services so to assure that the appropriate domain ontology is used to annotate the selected by the e-marketplaces semantic representation of a Web service. The market maker should secure the management and maintenance of underlying ontologies as they provide the right means for specifying such semantics by featuring logic-based representation languages [32. In order to allow meaningful matchmaking communication with the market has to take place on a semantic level. The usage of ontologies requires an introduction of reasoning functionalities to the e-marketplace. However, the mechanisms offered should be still tailored to both human user as well as machine to machine interactions as even while creating the e-marketplace for Semantic Web services, 
one has to remember to find an appropriate balance between computer automation and manual involvement. One cannot focus only and solely on automating transactions as we cannot forget that although the SWS are making their way, it is still a human that takes the last decision.

Another problem is associated with different levels of granularity of service description (coarse grained business services versus fine grained objects). We need to take into account that various service providers will describe their services (using of course the ontology provided by the e-marketplace) on the different level of abstraction. For one service provider, for example, the right level of abstraction will be providing their service as e.g. payment service (that implicitly will have such functionalities as customer verification, credit card number verification etc.), whereas other service providers will provide only atomic functionalities as customer verification, others may provide such functionalities as taking input from customer. The mechanism on the e-marketplace should be able to deal with this problem.

Discovery is the key functionality required in every e-marketplace. Clients must have a tool or mechanism that helps them find a service (or their composition) according to the specification provided. Useful solution here is to categorize the services and/or to perform a clustering over them [21. When it comes to traditional technical solutions the most popular are these using keyword matching algorithms and other text processing techniques. However, if we will consider the SWS e-marketplace, the algorithms need to operate on the semantic representation of a Web service. Therefore, the appropriate ontology-based SWS discovery mechanisms were developed [20] and are successfully utilized in various scenarios.

Personalization causes or tries to achieve the situation that every customer of the marketplace is treated uniquely. This mechanism, in its simplest form, is based on the system of user accounts. Such account keeps information about user, his personal data, interests, and other related to goods offered on the marketplace. It is very important that this data is secure. Issues with personal data can destroy trust which is later very hard to regain. The majority of marketplaces takes advantage of the system of user accounts. When user logs in, the personalized website opens for him, with tailored content as specified already in the user profile. To achieve the personalization the SWS filtering system needs to be implemented on the e-marketplace. It main aim is to gather the customer profiles (either the human user or acting on his behalf software agent) and using semantics-aware algorithms to filter the incoming stream of new SWS in order to find the relevant ones. The Semantic Web services clustering and filtering 22] are very helpful mechanism during the personalization of the offer as only services fulfilling user needs are then presented.

The issue of trust, which was until now only marginally mentioned, is in fact extremely important. Previous SOA solutions were successfully exploited internally in enterprises. These implementations were coupling only internal Web services or at most Web services only from a few trusted partners. With more trustworthy Web services, one will be more enthusiastic about using services of other parties in his own applications. Higher trust among parties effects in 
raised competition among service providers. Stronger competition means larger number of offered Web services. Moreover, in electronic commerce, trust between trading partners is considered to be just as important as in offline transactions and in some respects more important because of the nature of the channel.

Appropriate feedback mechanisms built into the e-marketplace, which allow participants to publicise their experiences, could improve the levels of trust between buyers and sellers and allow to update and the SWS characteristics. An exemplary functionality that could be applied here is provided by a service profiling 23. The profiling is used to determine the values of non-functional properties of services based on their execution history data. It allows to verify the correctness of the description provided by service providers. Moreover, as pinpointed by 24] in order to enable a true e-marketplace for services, there is a need for service clients to share their knowledge so as to help each other improve the quality of their decisions and learn from the previous interactions. In addition, e-marketplace requires ratings to attract new market opportunities and competition thus supporting dynamic real-time advertising [24].

The service composition is a mechanism that creates value added to SWS e-marketplace not only because it allows to fulfil clients' requirements (through composition of new applications), but also as new compositions become again services offered on the market. So in this way the number of available services is increasing all the time what makes the SWS e-marketplace more attractive to the users. With a use of sophisticated composition algorithms and methods 25. service chains may be created in order to fulfil complex user requirements. Carrying out the composition of SWS automatically is not a trivial issue. There are however many initiatives in this area. At first, enabling composite services has largely been an ad hoc, time-consuming and error prone process involving repetitive low-level programming. Then, the ontology-based frameworks for the automatic composition of Web services were introduced. A few different approaches/algorithms to automate service composition, most of them being an adaptation of planning algorithms, are used (e.g. ASG platform). Thus, there are still many issues that need to be investigated before the SWS composition algorithms will be taken fully advantage of by companies.

Semantic Web services e-marketplace must provide a possibility of contracting between customers and sellers. Web services are the kind of intangible good, whose main features, besides functionality, are in the performance and quality aspects. That is why the contracting infrastructure should be available on such a marketplace. Service level agreements should be defined with use of specific template, provided by the marketplace authorities/owners. The usage of formalized contracts prevents both sides (buyers and sellers) from any troubles in SLA interpretation in case of conflicts. An ideal situation would be if a marketplace was somehow connected with an infrastructure on which offered services are run. In that case it would be easy to monitor the performance of contracted services. Nevertheless, the storage of SLA instances in the marketplace repository is helpful, when we take into account that both buyers and sellers measure 
the performance of contracted services. When any violation is detected they can refer to the agreement stored in the marketpl platformository. The template of SLA should be constructed with regard to possible parameters to be measured, payment methods, discounts, fines, obligations and rights of both sides. The definition of such a template would be on a very general level, but to every domain of services there would be a need to create more detailed SLA templates. The contracting is heavily elaborated on in a number of publications and standards (e.g. 26] 27] and WSLA ).

The process that is an important point of every deal is terms/price negotiation. Semantic Web services are ideally designed to be negotiated. This results from the basic idea of Semantic Web, where intelligent agents can act on human's behalf. So, it is possible to equip both buyer and seller with preferences related to service delivery terms. The output of the negotiation process could be formed as an already mentioned service level agreement. An algorithm that does not give any advantage to buyer/seller implemented on the marketplace would enable the negotiation between agents. The negotiation strategies and detailed discussion on algorithms 28] are out of scope of this article.

Payment is another fundamental mechanism that every e-marketplace must have. We can divide payment services into two categories: buyer-seller based and third party based. First group of transactions causes the money flow between the buyer and seller without engagement of another body. Usually, when the agreed amount of money appears on the seller's account, the transaction is finalized. Second category covers these with third party intermediaries. Services such PayPal play a role of securers of the transactions. The latter category is more popular on international marketplaces, whose users come from many different countries. In case of SWS e-marketplace the payment mechanism is quite similar. However, it needs to be adapted to be used in the automatically carried out interactions.

Delivery of goods and deal tracking are crucial on the markets of tangible goods. They have a little bit different meaning on the market of Web services where the service may be either consumed using the Internet or the physical delivery needs to take place. When the deal is completed immediately, the thing to track or to be more specific - monitor, is the Web service execution itself (especially when it comes down to long-running Web services). On the other hand, when the delivery of a good takes more time and engages other parties (e.g. forwarding companies) it is very valuable for a customer to have the possibility of checking what is currently happening with the ordered item.

In consequence the following high level architecture of the SWS e-marketplace emerges as shown in figure 2. The main layer on which the e-marketplace is based is the adequate Semantic Web services representations along with the adequate reasoning possibility. The e-marketplace should support at least the following mechanisms that should operate on the semantics: publishing/registering, discovery and filtering, profiling, negotiation and contraction, composition, execution and monitoring, as well as trust and security and financial mechanisms.

${ }^{3}$ Web Service Level Agreements Project, http://www.research.ibm.com/wsla/ 


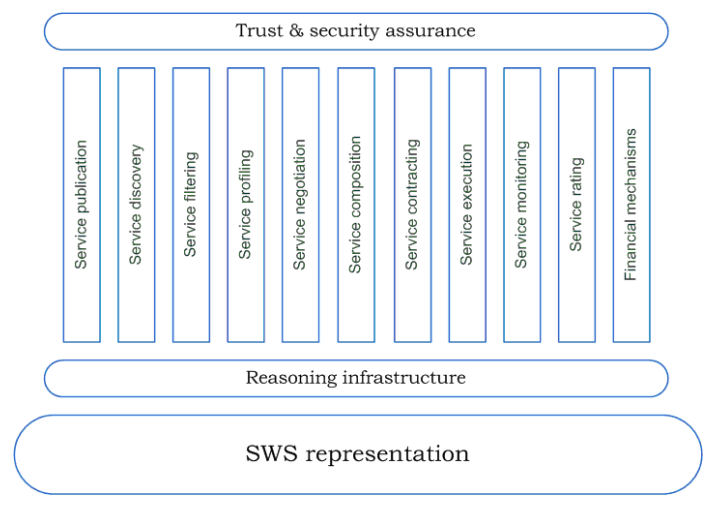

Fig. 2. Required functionalities of SWS e-marketplace

\section{SWS E-Marketplace Reference Model}

Within this section an abstract model of the SWS e-marketplace model is presented. We define SWS e-marketplace as an intermediary that allows service requesters and service providers to meet on an electronic platform that resides on the Internet infrastructure in order to exchange information about services (e.g. service description), conduct transactions online and adhere to other valueadded services offered by the intermediary. All artefacts and mechanisms on this e-marketplace are semantic-enabled. Let SWSEM denote Semantic Web services e-marketplace. It may be defined as follows:

$S W S E M=C, S P, S, M, P R, S A$

Where: $C$-denotes e-marketplace clients, for $i=1 \ldots k$. A service client is an entity that requests a service from a service provider (by invoking the service) and eventually consumes the service (sending request data and/or receiving the results). The term service client may be exchangeable used with terms service consumer and service requester.

The discussed e-marketplace is targeted at enterprises of all shapes and sizes or for the company internal purposes (especially those virtual ones having multiple branches in multiple locations to be used by employees/departments etc.). On the SWS e-marketplace organizations are treated as business users. Business users want to streamline or enhance their business processes by using best available pieces of software (in this case - Semantic Web services). They manifest their needs in a form of client profile. Within our model we envision a presence of software agents acting on behalf of clients. Such an agent must be provided with the profile of a user, negotiation policies and constraints defining acceptable terms of service provisioning.

$S P$ - denotes service providers, for $i=1 \ldots p$. Service providers may be divided into two groups ASP (atomic service providers) and CSP (composite service providers). Some providers may belong to two groups as they may offer both atomic as well as composite services. One may say, that the special case of CSP 
is an e-marketplace itself as it performs service composition and may offer to clients composite services. Service providers are also represented on the SWS e-marketplace using appropriate profile informing about the contact details as well as Semantic Web services they provide and their quality. Application developers are sophisticated participants of SWS e-marketplace. They are familiar with technical aspects of SWS and exactly know what they need. They are prepared to describe precisely which SWS is needed in their application. Developers need rather one-time solutions from the e-marketplace, in order to finish and run their application. However, they may update their application if a better service appears.

Brokers have more transactional power than single participants. We can distinguish brokers acting on providers' and customers' side. More popular are customers' side brokers. They may negotiate better terms with providers as they usually buy. Next, brokers may resell these services with some margin. Providers' side brokers are rare, but may appear when powerful customer emerges on the market and his requests may not be served by single providers. However, it should not be a case in the domain of SWS where only the scalability of computational infrastructure constitutes the limit.

$S$ - denotes a set of services (both atomic and composite ones, also those composed by the e-marketplace itself), for $i=1 \ldots s$. Atomic service is a service that does not rely on other services during execution. In case of composite services it is quite the opposite - they do rely on other services during their execution. Please note, that for the e-marketplace as well as clients there is not much difference whether it is a composite or atomic service that is offered by the service provider. Both services are black boxes and we cannot see their internal structure (the internal logic of the composition) as it is hidden to avoid being copied by other parties and loosing the competitive advantage by the service provider. However, the difference lies in that the composite service may be improved by relying on different services during execution. The improvement of an atomic service requires changing the implementation of the service (new algorithms, new logic, new hardware etc.). The repository that would store the services description on both the functional as well as non-functional properties is also a part of the SWS model. On the high level it is not important which particular language is used to describe a service. However, the market maker needs to ensure that all services are described using the same ontology as well as that the domain ontologies are known to e-marketplace actors.

The services offered on SWS e-marketplace may be divided in two groups: information services and real world services. The difference between these two types lays in the nature of results obtained as a result of their execution. In case of information services a piece of information is both an effect and an output of a service execution. As an example may serve services responsible for some computation and all those that only operate on virtual objects. In case of real world services SWS are only the interface to real functionality that must be provided in a real world. So, we can use SWS that is an interface to ordering a book, but as an output we obtain a digital confirmation of a purchase, but the 
effect is the real book that is shipped few days later. In our understanding of Semantic Web services we pay special attention to their description. We advocate that the description of services offered on the SWS e-marketplace must be as informative as possible and should cover aspects of their functionality as well as quality.

$M$ - denotes mechanisms (functionalities offered by the marketplace) and tools provided to clients and service providers (for example: composition, selection, profiling, contracting, monitoring); for $i=1 \ldots m$. They were already discussed within the previous section.

$P R$ - denotes participation rules and a business model - all the rules that are to organize the provisioning, supply and demand matching process, for $i=1 \ldots r$. E-marketplaces should provide their users with institutional infrastructure that encompasses issues related to contract law, dispute resolution, and intellectual property protection. These business rules must be enforced and monitored on the e-marketplace. These aspects, as is shown within the next section, are disregarded by most if not all of the current initiatives.

$S A$ - supporting artefacts - all artefacts - SLAs, domain ontologies, extended OWL-S etc. This relates to the indispensable data structures, ontologies and languages used in the e-marketplace to process information about services, enable transactions and support all the functionalities offered by the e-marketplace.

\section{Comparison of SWS Frameworks}

In order to compare proposed model with current initiatives in the Web services provisioning, please take a look at the figure 3. This table presents the most important aspects and their coverage by selected (S)WS market models.

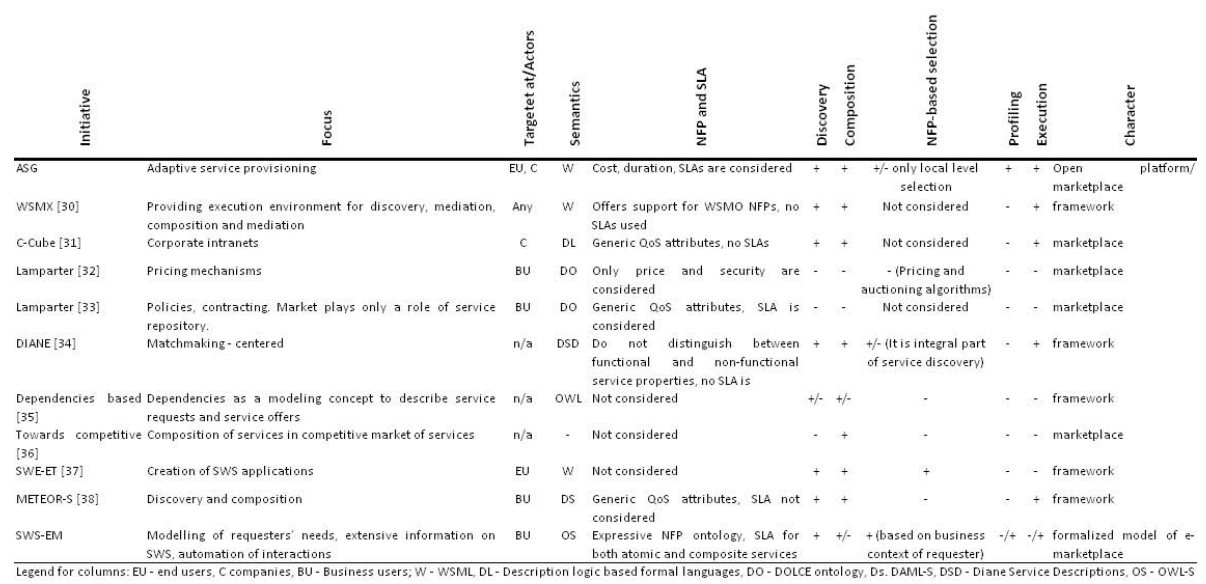

Fig. 3. Comparison of SWS frameworks 
The important conclusion is that these approaches have usually different research goals. Most of them are composition- or discovery-oriented, whereas some address more economical issues of SWS provisioning. Our SWS-EM model is more economically oriented, but we do not ignore the technical aspects. Therefore, some elements are marked $+/$ - which means that the reference model considers them, but our research goals and detailed analysis are now aimed at missing aspects.

What is also interesting is that most approaches use their own languages for describing services. This may be the reason that they lack generality and their potential usage by wider communities is doubtful. Recent research [40] clearly shows that the most popular semantic language is OWL. Therefore, the use of OWL based service descriptions seems to be a right choice.

As also may be concluded from the table, the described functionalities of the Semantic Web services e-marketplace do not have to be implemented from scratch. The enumerated interactions along with several algorithms implementing them [39], are already applied in business scenarios [29] or DIP. However in order for the SWS marketplace to be successful lessons learned need to be taken into account. In the first generation of Web services the target user was a programmer. However, right now the target users of the e-marketplaces (and also SWS e-marketplace) are domain experts, consultants and business specialists implementing business processes through service composition [14]. The current description stack and interactions are tailored to the needs of the developers and not the business people. WS and SWS should not be perceived only as programmable components. They are also a kind of commercial commodity provided via the Internet with a remarkable trait of combining them in functional workflows fulfilling the user's need of resolving their non-trivial problems. The mechanisms of the SWS e-marketplace should take advantage of the already existing achievements in the area of SWS interactions, however all the mechanisms need to be adjusted to the new target user and extended with business aspects.

\section{Conclusions and Future Work}

The idea of the SWS e-marketplace presented in this article was developed as a result of research carried out in the area of SOA and Web services technology. Fully-fledged SWS e-marketplaces should provide a large set of service support and other functionalities, e.g.:

- providing to its participants a unified view of services, standard business terminology and detailed composite services descriptions,

- a comprehensive range of functionalities supporting trade, negotiation, financial settlements, service certification and quality assurance, rating services and service metrics and manage the negotiation and enforcement of SLAs,

- the market maker takes the responsibility of marketplace administration and performs maintenance tasks to ensure its quality and reliability.

The SWS e-marketplaces should be created with the cutting-edge information technology. However, to be truly successful, they must exceed their technological 
roots and offer a support to the relationship management, personalization, oneto-one marketing as well as trust and security.

Solutions available at the moment hardly meet the enumerated postulates. Current e-marketplaces are basically simple websites storing information on available services. There is a place for marketplace with described features as it would create a brand new value for the users. The amount of features that the marketplace is to be equipped with, allows for effective use, thus creating a feedback for the new potential users. This statement is made upon the experiences of marketplaces of the class of eBay.com and community portals that share some characteristics with the marketplace.

We have to clearly state that the mechanisms of the featured framework can have some issues that can spoil all the benefits enumerated. We are aware of the obstacles and challenges that one has to tackle when creating the e-marketplace for Semantic Web services. The future work is to investigate in details the mechanisms presented in this article, elaborate on the issues of trust and security on the SWS e-marketplace and then provide a prototypical implementation of the proposed solution.

The current trends in the software domain should be closely watched. Currently we observe also a change in the way of the software distribution and provisioning. There is a shift towards the software as a service model and services and their non-functional characteristics are placed in the centre of interest. In this situation the mechanisms offered by the SWS e-marketplace will provide users with a required support on the global market of the future.

\section{References}

1. Hepp, M., Leymann, F., Domingue, J., Wahler, A., Fensel, D.: Semantic Business Process Management: A Vision Towards Using Semantic Web Services for Business Process Management. In: Proceedings of the IEEE ICEBE 2005, Beijing, China, October 18-20, pp. 535-540 (2005)

2. Lamparter, S., Agarwal, S.: Specification of Policies for Automatic Negotiations of Web Services. In: Hendler, L.K.A.T.F.A.J. (ed.) Semantic Web and Policy Workshop, held in conjunction with the 4th International Semantic Web Conference, Galway, Ireland (2005)

3. Papazoglou, M.P., Georgakopoulos, D.: Service-oriented computing. Introduction to the Communications of the ACM 46(10) (October 2003)

4. Papazoglou, M.P., Traverso, P., Dustdar, S., Leymann, F.: Service-Oriented Computing Research Roadmap. European Union Information Society Technologies (IST), Directorate D

5. Li, L., Horrocks, I.: A software framework for matchmaking based on semantic web technology. In: Proceedings of the 12th International Conference on the World Wide Web, Budapest, Hungary (May 2003)

6. Bussler, C., Maedche, A., Fensel, D.: A Conceptual Architecture for Semantic Web Enabled Web services. ACM Special Interest Group on Management of Data 31(4) (December 2002)

7. Deng, S., Wu, Z., Li, Y.: ASCEND: a framework for automatic service composition and execution in dynamic environment. In: Proceedings of International Conference Systems, Man and Cybernetics, pp. 3457-3461 (2004) 
8. Bakos, J.Y.: Reducing buyer search costs: Implications for electronic marketplaces. Management Science 43(12), 1676-1692 (1997)

9. Hadaya, P.: Determinants of the future level of use of electronic marketpalces: The case of Canadian firms. Electronic Commerce Research 6(2), 173-185

10. Raisch, W.D.: The eMarketplace: Strategies for success in B2B ecommerce. McGraw Hill, New York (2001)

11. Adams, J., Koushik, S., Vasudeva, G., Galambos, G.: Patterns for e-business - a strategy for reuse (2001)

12. Feldman, S.: E-business: Electronic Marketplaces. IEEE Internet Computing, (4) 93-95 (2000)

13. Grey, W., Olavson, T., Shi, D.: The role of e-marketplaces in relationship-based supply chains: a survey. IBM Systems Journal 44(1) (2005)

14. Hidalga, A.N., Zhao, L., Falcone-Sampaio, P.R.: Leveraging e-marketplaces models for Web service-based application development. In: Pages-Casas, L. (ed.) Web services, vol. VII (2006)

15. Mueller, I., Braun, P., Rossak, W.: Integrating Mobile Agent Technology into an emarketplace solution: The InterMarket Marketplace: Friedrich-Schiller-University Jena (2002)

16. Chiu, D.K.W., Poon, J.K.M., Lam, W.C., Tse, C.Y., Su, W.H.T., Poon, W.S.: How ontologies can help in an e-marketplace. In: 13th European Conference on Information Systems, Information Systems in Rapidly Changing Economy (ECIS 2005), Regensburg, Germany, May 26-28, 2005) (2005)

17. Lamparter, S., Schnizler, B.: Trading services in ontology-driven markets. In: The Proceedings of the 2006 ACM symposium on Applied computing, pp. 1679-1683. ACM Press, New York (2006)

18. Li, Z., Zhao, H., Ramanathan, S.: Pricing web services for optimizing resource allocation - an implementation scheme. In: Web 2003, Seattle (2003)

19. Shmueli, O.: Architectures For Internal Web Services Deployment. In: Proceedings of the 27th VLDB Conference, Roma, Italy (2001)

20. Liu, C., Peng, Y., Chen, J.: Web Services Description Ontology-Based Service Discovery Model. In: IEEE/WIC/ACM International Conference on Web Intelligence (WI 2006 Main Conference Proceedings) (WI 2006), pp. 633-636 (2006)

21. Abramowicz, W., Haniewicz, K., Kaczmarek, M., Zyskowski, D.: Architecture for Web services filtering and clustering. In: The Proceedings of ICIW 2007, IEEE 2007 (2007)

22. Abramowicz, W., et al.: Application-oriented Web Services Filtering. In: The Proceedings of International Conference on Next Generation Web Services Practices, pp. 63-68. IEEE, Los Alamitos (2005)

23. Abramowicz, W., et al.: Architecture for Service Profiling. In: Castellanos, M., Yang, J. (eds.) Proceedings of 2006 IEEE Services Computing Workshops (SCW 2006), pp. 121-127. IEEE Press, Los Alamitos (2006)

24. Maximilien, E.M., Singh, M.P.: Conceptual Model of Web services Reputation. SIGMOD Record (2002)

25. Akkiraju, R., et al.: Combining planning with semantic matching to achieve web service composition. In: 4th International Conference on Web Services, ICWS 2006 (2006)

26. Lamparter, S., Luckner, S., Mutschler, S.: Formal Specification of Web Service Contracts for Automated Contracting and Monitoring. In: The Proceedings of the 40th Hawaii International Conference on System Sciences. IEEE, Los Alamitos (2007) 
27. Oldham, N., Verma, K., Sheth, A., Hakimpour, F.: Semantic WS-Agreement Partner Selection. In: Proc. of the 15th Int. WWW Conf., Edinburgh, UK (2006)

28. Vivying, S.Y., Cheng, V.S.Y., Hung, P.C.K., Chiu, D.K.W.: Enabling Web Services Policy Negotiation with Privacy preserved using XACML, HICSS. In: 40th Annual Hawaii International Conference on System Sciences (HICSS 2007), p. 33 (2007)

29. Kuropka, D., Weske, M.: Implementing a semantic service provision platform. In: Concepts and Experiences, Wirtschaftsinformatik, vol. 1, pp. 16-24 (2008)

30. Haller, A., Cimpian, E., Mocan, A., Oren, E., Bussler, C.: WSMX - A Semantic Service-Oriented Architecture. In: Proceedings of the IEEE International Conference on Web Services. IEEE Computer Society, Los Alamitos (2005)

31. Canfora, G., Corte, P., De Nigro, A., Desideri, D., Di Penta, M., Esposito, R., Falanga, A., Renna, G., Scognamiglio, R., Torelli, F., Villani, M., Zampognaro, P.: The C-Cube framework: developing autonomic applications through web services. SIGSOFT Softw. Eng. Notes 30, 1-6 (2005)

32. Lamparter, S., Schnizler, B.: Trading services in ontology-driven markets. In: Proceedings of the 2006 ACM symposium on Applied computing, Dijon, France. ACM, New York (2005)

33. Lamparter, S.: Policy-based Contracting in Semantic Web Service Markets. Karlsruhe, Univeristy of Karlsruhe (2007)

34. Kuster, W., Koenig-Ries, B., Stern, M., Klein, M.: DIANE: an integrated approach to automated service discovery, matchmaking and composition. In: Proceedings of the 16th international conference on World Wide Web, Banff, Alberta, Canada. ACM Press, New York (2007)

35. Tolksdorf, R., Bizer, C., Heese, R.: A Web Service Market Model based on Dependencies. In: The Twelfth International World Wide Web Conference (WWW 2003) (Posters), Budapest, Hungary (2003)

36. Cheng, S., Chang, C., Zhang, L., Kim, T.-H.: Towards Competitive Web Service Market. In: Proceedings of the 11th IEEE International Workshop on Future Trends of Distributed Computing Systems. IEEE Computer Society, Los Alamitos (2007)

37. Brambilla, M., Ceri, M., Facca, F., Celino, I., Cerizza, D., Valle, E.D.: Model-driven design and development of semantic Web service applications. ACM Trans. Interet Technol. 8(3)

38. Verma, K., Sivashanmugam, K., Sheth, A., Patil, A., Oundhakar, S., Miller, J.: METEOR-S WSDI: A Scalable P2P Infrastructure of Registries for Semantic Publication and Discovery of Web Services. Inf. Technol. and Management 6, 17-39 (2005)

39. Abamowicz, W., et al.: Automatic Web services interactions - requirements, challenges and limits from the F-WebS system perspective. In: The Proceedings of International Conference on Next generation Web Services Practices, IEEE 2006 (2006)

40. Cardoso, J.: The Semantic Web Vision: Where Are We? IEEE Intelligent Systems 22, 84-88 (2005)

41. Barros, A., Dumas, M., Bruza, P.: The move to Web services ecosystem. BPTrends (2005) 